\title{
Can Canadians afford the new Canada's Food Guide? Assessing Barriers and Challenges
}

\author{
Dr. Sylvain Charlebois ${ }^{1}$, Meghan Smook ${ }^{2}$, Brenda Nyambura Wambui ${ }^{3}$, Dr. Simon Somogyi ${ }^{4}$, Dr. Megan Racey ${ }^{5}$, \\ Don Fiander ${ }^{6}$, Janet Music ${ }^{7} \&$ Isabelle Caron ${ }^{7}$ \\ ${ }^{1}$ Agrifood Analytics Lab, Faculty of Agriculture, Dalhousie University, Faculty of Management, Kenneth C. \\ Rowe Management Building, Room 3059, Canada \\ ${ }^{2}$ School of Public Administration, Faculty of Management, Dalhousie University, Canada \\ ${ }^{3}$ Department of Economics, Faculty of Science, Dalhousie University, Canada \\ ${ }^{4}$ Chair of the Arrell Food Institute, School of Hospitality, Food \& Tourism Management, and College of \\ Business and Economics, Canada \\ ${ }^{5}$ Department of Human Health and Nutritional Sciences, University of Guelph, Canada \\ ${ }^{6}$ DalAnalytics, Dalhousie University, Canada \\ ${ }^{7}$ Faculty of Management, Dalhousie University, Canada \\ Correspondence: Sylvain Charlebois, 6100 University Avenue, PO Box 15000, Halifax NS, Canada B3H 4R2, \\ Canada. E-mail: sylvain.charlebois@dal.ca
}

Received: October 26, 2021

doi:10.5539/jfr.v10n6p22
Accepted: November 25, $2021 \quad$ Online Published: November 26, 2021

URL: https://doi.org/10.5539/jfr.v10n6p22

\begin{abstract}
The objective of this study was to investigate Canadian's awareness and use of Canada's Food Guide and to explore barriers to adopting the recommendations. We also conducted a cost analysis to measure the affordability of the 2019 Food Guide compared to the previous version. Although $74 \%$ of Canadians were aware of the new Food Guide, it ranked low as a preferred source of information; as well, affordability is a top concern when implementing the recommendations. However, eating based on the 2019 Food Guide is more affordable than the 2007 version at food prices in either year. These results bring into question the influence Food Guides have on population's health behaviour.
\end{abstract}

Keywords: Canada's Food Guide, food security, cost analysis, food policy

\section{Introduction}

Internationally, governments use food based dietary guidelines (FBDG) to recommend and promote diets that encourage healthy populations and prevent the incidence of non-communicable diseases (Painter, Rah, \& Lee, 2002). The WHO acknowledges the role of these guidelines as instruments that influence "a wide range of food and nutrition, health, agriculture, and nutrition education policies and programs" (Food-based dietary guidelines, 2019). FBDG often are presented as visual depictions of a balanced diet and are sometimes referred to as food guides, or food pyramids. FBDG can serve as strategic policy documents that not only help governments encourage healthy eating behaviours among their citizens, but are also key education documents for teaching youth about healthy eating, and are used to create balanced meals in institutions such as hospitals and long-term care homes.

Since the first publication of Canada's Food Guide (CFG), then known as "Canada's official Food Rules" in 1942, the Government of Canada has used the document as a policy instrument to help shape Canadians' perceptions of food and influence food consumption behaviours (Canada - Food Safety \& Supply; Health Canada Canada's Food Guides from 1942 to 1992). Canada's Official Food Rules were intended to respond to food shortages that resulted from the Second World War. They emphasized "wartime food rationing, while endeavoring to prevent nutritional deficiencies and improve the health of Canadians" (Health Canada Canada's Food Guides from 1942 to 1992). Since then, there have been multiple updates and changes to the Food Guide, including the addition of food groups in 1961 and the consolidation of these food groups in 1977. The 2007 version of CFG was published in "multiple languages to reflect changing population demographics" and was 
also adapted to meet the unique cultural needs of First Nations, Inuit and Metis populations (Slater \& Mudryj, 2018).

The Canadian federal government published the most recent edition of CFG on 22 January 2019 (Canada's Food Guide, 2019). The newest version of the Food Guide saw substantial changes, such as the removal of food groups, and encourages Canadians to eat a variety of healthy foods each day, including plenty of vegetables and fruit, protein foods, and whole grains (Health Canada, 2018). Beyond educating Canadians on what to eat, the new CFG also provides advice on how to eat. It encourages Canadians to be mindful of their eating habits, to cook at home, to eat meals with others, and to enjoy their food (Canada's Food Guide, 2019). It is now the most downloaded document, after tax forms, from the Government of Canada's website.

\subsection{Use of Food Guide for Nutrition Information}

However, research has called in to question the impact that food based dietary guidelines have on consumer behaviours. While most Canadians are familiar with CFG and it may be a frequently downloaded document, few use it to guide their healthy eating decisions (Charlebois, McCormick, \& Juhasz, 2016; Slater \& Mudryj, 2018). This corresponds to similar international studies which found that consumers are more likely to be aware of and understand food based dietary guidelines than they are to use food based dietary guidelines (Brown et al., 2011).

Based on data from the 2012 Canadian Community Health Survey, most respondents did not use any sources to consult on healthy eating recommendations. If respondents did seek out nutrition-related advice, they did so through means of general research and seeking the advice of family and friends (Slater \& Mudryj, 2018). This is not surprising as the Food Guide exists among a sea of healthy eating information available online (Slater \& Mudryj, 2018).

\subsection{Motivation for Healthy Eating}

While consumers may show a high level of comprehension and understand the importance of FBDG, consumer food decisions are not made based on nutritional merit or the presentation of evidence-based information alone. Many approaches to dietary change include nutrition education and the provision of information; however, these often have limited and short-lived effects at best (Brug, 2008; Pelletier \& Laska, 2012). People's motivations for healthy eating are diverse and complex and include taste preferences, intention, convenience, time, familiarity, and price. In fact, $40 \%$ of Americans and 57\% of Europeans indicated that they would rarely or never compromise on taste to improve the healthfulness of their diets (Brug, 2008). In addition, people must have the confidence, skills, and abilities to put these recommendations into practice (Brug, 2008). Without these abilities, consumers will be unable to incorporate recommended foods and behaviours into their daily lives. This begs the question about the applicability and usefulness of FBDG, given the complex factors that influence eating and the wealth of online knowledge that can be easily accessed by consumers.

\subsection{Implications for Food Security}

Beyond these internal and individual factors, one of the most influential external factors affecting food choice is the environment in which people live, which closely relates to the availability, affordability, and accessibility of food and ultimately, a family's level of food security (Brug, 2008; Love, Maunder, \& Green, 2008). According to the Food and Agricultural Organization, food security is "when all people, at all times, have physical and economic access to sufficient, safe and nutritious food to meet their dietary needs and food preferences for an active and healthy life (The Rome Declaration, 1996)." Approximately one in eight Canadian households is food insecure, amounting to over 4 million Canadians, including 1.15 million children (Tarasuk, 2005). Research shows that those living in food insecure and low-income households struggle to meet recommendations and eat diets that align with FBDG resulting in poor dietary quality and heightened nutritional vulnerability (Breland et al., 2013; Gregório et al., 2018; Loopstra \& Tarasuk, 2012). Even though Canadian governments have recognized food and income as important determinants of health, there has been little advancement of effective policies to mitigate household food insecurity (Loopstra \& Tarasuk, 2012). The newly released CFG was also criticized for lacking in considerations regarding affordability and cost when the recommendations were developed.

Based on the efforts to develop FBDG and their potential for affecting consumer behaviour and population eating habits, this study had two distinct parts, with separate research questions. Part I of this study investigated Canadians' perceptions and awareness of Canada's Food Guide through an online survey. This survey determined Canadian consumers' awareness, perceived relevance, and use of Canada's Food Guide as well as any barriers consumers may perceive in adopting the recommendations of Canada's Food Guide. Secondly, Part II of the study investigated the affordability of the guide based on plates that would meet the recommendations. 
This was also compared to the 2007 Food Guide to determine the cost to consumers meeting the new guidelines and its impact on food security since affordability is a primary factor affecting a family's overall food security.

\section{Methods}

\subsection{Part I: Development, Validation, and Administration of Online Survey}

Part I of this study included the use of an online survey to determine Canadians' awareness, perceived relevance, and use of CFG, including any perceived barriers in adopting the recommendations. The study and questionnaire were approved by both Dalhousie University's and the University of Guelph's Research Ethics Boards.

Survey questions were developed based on past similar risk perception studies related to nutrition and food. The survey instrument was structured into five main parts (see Appendix A) and is briefly summarized below.

1. Dietary preferences and restrictions.

2. Sources of information where consumers gather nutritional information.

3. Awareness and use of the Food Guide within recent months.

4. Barriers to implementing recommendations from the Food Guide.

5. Perceptions about the practicality/applicability of the Food Guide.

To ensure the survey was effective and questions were well worded and understood correctly, a pretest was conducted. The pretest involved 52 respondents who went through the survey and commented on issues of clarity and the general understanding of the questions.

The survey was conducted over two days in February 2019 through an accessible e-platform, Qualtrics.com, in both French and English to capture a full and accurate reflection of the Canadian market. It is important to note the survey was conducted more than eight weeks after the new guide was released, and it was no longer mentioned in the media regularly. To minimize self-selection biases, no references to the goals or purpose of the study were made in the letter to respondents. The survey was administered to 1017 adult Canadians to determine how they perceived CFG and its relevancy to their personal food choices. All respondents had to be 18 years of age or older and have lived in Canada for at least twelve months. Consent was obtained by all respondents through the first question of the survey, although withdrawal from the survey was possible throughout all stages.

The completion rate of the survey was $97 \%$, and respondents took 4.5 minutes on average to complete the survey. After data were collected, the participants were thanked and debriefed. Based on the sampling design, the margin of error is $3.1 \%$, or 19 times out of 20 . The performance of this survey is consistent with other similar surveys on perceptions in food (Barcellos et al. 2015).

\subsection{Part II: Cost of Food Comparisons and Affordability of CFG}

For Part II of the study, we compared the affordability of the 2019 CFG to the previous 2007 version. The aim was to access the affordability of both guides. Calculations did not eliminate the influence of food inflation, but rather looked at changes in the physical quantities now deemed important for health in 2019 as compared to 2007. In doing so, our evaluation reflects the nature of the market and economic conditions which consumers had to deal with. Extracting any anomalies from food prices between 2007 and 2019 would have been challenging, if not impossible.

To compare affordability between guides, we created 'plates' of food that met the guidelines and recommendations for the 2007 Food Guide and the 2019 Food Guide, since both Food Guides recommended different proportions of foods and different types of food (see Table 1 for plate composition, Table 2 for 2007 information and conversion details, and Table 3 for 2019 information and conversion details). Information and prices are based on StatsCan recommended food basket. For example, based on the 2007 CFG, a male aged 19-50 years old consumes 22 food group servings per day which consists of three servings of meat and alternatives (13.5\%), two servings of milk and alternatives (9\%), eight servings of grains (36.5\%), and approximately nine servings of vegetables and fruit (40\%). In contrast, based on the 2019 CFG, a 'plate' for a male aged $19-50$ years old would consist of $25 \%$ whole grain foods, $25 \%$ protein foods, and $50 \%$ vegetables and fruit. 
Table 1. Plate composition

\begin{tabular}{|c|c|c|c|c|c|c|c|c|c|c|c|c|}
\hline & M2007 & MIN & MAX & D2007 & MIN & MAX & M2018 & MIN & MAX & D2018 & MIN & MAX \\
\hline MEAT & 0.851475 & 0.4275 & 1.55175 & 0.828525 & 0.432 & 1.5795 & 1.298 & 0.531 & 2.25975 & 1.309275 & 0.564 & 2.42325 \\
\hline EGGS & 0.411667 & 0.411667 & 0.411667 & 0.411667 & 0.411667 & 0.411667 & 0.503333 & 0.503333 & 0.503333 & 0.516667 & 0.516667 & 0.516667 \\
\hline BAKED BEANS & 0.40892 & 0.40892 & 0.40892 & 0.395729 & 0.395729 & 0.395729 & 0.540829 & 0.540829 & 0.540829 & 0.523241 & 0.523241 & 0.523241 \\
\hline CANNED SALMON & 1.179577 & 1.179577 & 1.179577 & 1.112676 & 1.112676 & 1.112676 & 1.588028 & 1.588028 & $\begin{array}{l}3.588028 \\
\end{array}$ & 1.795775 & 1.795775 & 1.795775 \\
\hline Average Meat & 0.71291 & 0.606916 & 0.887978 & 0.687149 & 0.588018 & 0.874893 & 0.982548 & 0.790798 & 1.222985 & 1.036239 & 0.849921 & 1.314733 \\
\hline CHEESE & 0.556 & 0.556 & 0.556 & 0.552 & 0.552 & 0.552 & 0.526 & 0.526 & 0.526 & 0.514 & 0.514 & 0.514 \\
\hline EVAP. MILK & 0.512987 & 0.512987 & 0.512987 & 0.496753 & 0.496753 & 0.496753 & 0.63961 & 0.63961 & 0.63961 & 0.594156 & 0.594156 & 0.594156 \\
\hline Average Dairy & 0.534494 & 0.534494 & 0.534494 & 0.524377 & 0.524377 & 0.524377 & 0.582805 & 0.582805 & 0.582805 & 0.554078 & 0.554078 & 0.554078 \\
\hline Average (2019 FG) & 0.560089 & 0.499522 & 0.660129 & 0.542479 & 0.485832 & 0.649761 & 0.727972 & 0.6184 & 0.865364 & 0.750445 & 0.643977 & 0.909584 \\
\hline BREAD & 0.106296 & 0.106296 & 0.106296 & 0.113037 & 0.113037 & 0.113037 & 0.144667 & 0.144667 & 0.144667 & 0.145185 & 0.145185 & 0.145185 \\
\hline MACARONI & 0.0945 & 0.0945 & 0.0945 & 0.1062 & 0.1062 & 0.1062 & 0.1233 & 0.1233 & 0.1233 & 0.1341 & 0.1341 & 0.1341 \\
\hline CORN FLAKES & 0.174667 & 0.174667 & 0.174667 & 0.173333 & 0.173333 & 0.173333 & 0.233778 & 0.233778 & 0.233778 & 0.254222 & 0.254222 & 0.254222 \\
\hline Average Grain & 0.125154 & 0.125154 & 0.125154 & 0.130857 & 0.130857 & 0.130857 & 0.167248 & 0.167248 & 0.167248 & 0.177836 & 0.177836 & 0.177836 \\
\hline$F \& V$ & 0.2772 & 0.109375 & 0.644 & 0.224613 & 0.09625 & 0.602 & 0.321563 & 0.140875 & 0.746375 & 0.327775 & 0.1365 & 0.772625 \\
\hline POTATOES & 0.082682 & 0.082682 & 0.082682 & 0.072274 & 0.072274 & 0.072274 & 0.148789 & 0.148789 & 0.148789 & 0.156305 & 0.156305 & 0.156305 \\
\hline CANNED TOMATOES & 0.199435 & 0.199435 & 0.199435 & 0.201005 & 0.201005 & 0.201005 & 0.227701 & 0.227701 & 0.227701 & 0.233982 & 0.233982 & 0.233982 \\
\hline Average F\&V & 0.186439 & 0.130497 & 0.308705 & 0.165964 & 0.123176 & 0.29176 & 0.232684 & 0.172455 & 0.374288 & 0.239354 & 0.175596 & 0.387637 \\
\hline JUICE & 0.2075 & 0.20125 & 0.21375 & 0.20875 & 0.19625 & 0.22125 & 0.28125 & 0.2475 & 0.315 & 0.276875 & 0.25625 & 0.2975 \\
\hline ORANGE JUICE & 0.344669 & 0.344669 & 0.344669 & 0.338235 & 0.338235 & 0.338235 & 0.375919 & 0.375919 & 0.375919 & 0.382353 & 0.382353 & 0.382353 \\
\hline Average F\&V w/ juice & 0.222297 & 0.187482 & 0.296907 & 0.208975 & 0.180803 & 0.286953 & 0.271044 & 0.228157 & 0.362757 & 0.275458 & 0.233078 & 0.368553 \\
\hline
\end{tabular}


Table 2. Plate details, weighted data, family composition and conversions for 2007 guide

\begin{tabular}{|c|c|c|c|c|c|c|c|c|c|c|c|c|}
\hline & M2007 & MIN & MAX & D2007 & MIN & MAX & M2018 & MIN & MAX & D2018 & MIN & MAX \\
\hline Average Meat & 0.71291 & 0.606916 & 0.887978 & 0.687149 & 0.588018 & 0.874893 & 0.982548 & 0.790798 & 1.222985 & 1.036239 & 0.849921 & 1.314733 \\
\hline Average Dairy & 0.534494 & 0.534494 & 0.534494 & 0.524377 & 0.524377 & 0.524377 & 0.582805 & 0.582805 & 0.582805 & 0.554078 & 0.554078 & 0.554078 \\
\hline Average Protein $(2019 \mathrm{~F}$ & 0.560089 & 0.499522 & 0.660129 & 0.542479 & 0.485832 & 0.649761 & 0.727972 & 0.6184 & 0.865364 & 0.750445 & 0.643977 & 0.909584 \\
\hline Average Grain & 0.125154 & 0.125154 & 0.125154 & 0.130857 & 0.130857 & 0.130857 & 0.167248 & 0.167248 & 0.167248 & 0.177836 & 0.177836 & 0.177836 \\
\hline Average F\&V & 0.186439 & 0.130497 & 0.308705 & 0.165964 & 0.123176 & 0.29176 & 0.232684 & 0.172455 & 0.374288 & 0.239354 & 0.175596 & 0.387637 \\
\hline \multirow[t]{4}{*}{ Average $F \& V$ w/ juice } & 0.222297 & 0.187482 & 0.296907 & 0.208975 & 0.180803 & 0.286953 & 0.271044 & 0.228157 & 0.362757 & 0.275458 & 0.233078 & $\begin{array}{ll}30.368553 \\
\end{array}$ \\
\hline & & & & & & & & & & & & \\
\hline & & & & & & & & & & & & \\
\hline & May-07 & & & Dec-07 & & & May-18 & & & Dec-18 & & \\
\hline Male (19-50) & average $r$ & $\min$ & $\max$ & average & $\min$ & $\max$ & average & $\min$ & $\max$ & average & $\min$ & $\max$ \\
\hline f\&v w/ juice & 2.000674 & 1.687339 & 2.672164 & 1.880779 & 1.627226 & 2.582576 & 2.439398 & 2.053411 & 3.264811 & 2.479123 & \begin{tabular}{|l|}
3 \\
\end{tabular} .097703 & 3.316978 \\
\hline grain & 1.001235 & 1.001235 & 1.001235 & 1.046854 & 1.046854 & 1.046854 & 1.337985 & 1.337985 & 1.337985 & 1.422686 & 1.422686 & 1.422686 \\
\hline \multirow{2}{*}{$\begin{array}{l}\text { milk } \\
\text { meat }\end{array}$} & 1.068987 & 1.068987 & 1.068987 & 1.048753 & 1.048753 & 1.048753 & $3 \quad 1.16561$ & 1.16561 & 1.16561 & 1.108156 & 1.108156 & 1.108156 \\
\hline & 2.138729 & 1.820748 & 2.663935 & 2.061447 & 1.764054 & \begin{tabular}{|l}
2.624679 \\
\end{tabular} & 2.947643 & 2.372393 & 3.668955 & 3.108718 & $\begin{array}{l}3.549762 \\
\end{array}$ & 3.944199 \\
\hline Total Cost (Daily) & 6.209625 & 5.578308 & 7.406321 & 6.037834 & 5.486887 & 7.302862 & 7.890637 & 6.929399 & 9.437362 & 8.118683 & 7.178307 & 9.792019 \\
\hline f\&v w/ juice & 2.445268 & 2.062303 & 3.265978 & 2.29873 & 1.988832 & 3.156482 & 2.981487 & 2.509724 & 3.990324 & 3.030039 & 2.563859 & 4.054084 \\
\hline grain & 0.688349 & 0.688349 & 0.688349 & 0.719712 & 0.719712 & 0.719712 & 0.919865 & 0.919865 & 0.919865 & 0.978097 & 0.978097 & 0.978097 \\
\hline protein & 3.430359 & 3.138876 & 3.911798 & 3.331696 & 3.059085 & 3.847991 & 4.30472 & 3.777408 & 4.965923 & 4.373373 & 3.860996 & 5.13923 \\
\hline Total Cost (Daily) & 6.563976 & 5.889528 & 7.866125 & 6.350138 & 5.767629 & 7.724185 & 8.206072 & 7.206997 & 9.876112 & 8.381508 & 7.402952 & 10.17141 \\
\hline & Mav-07 & & & Dec-07 & & & Mav-18 & & & Dec-18 & & \\
\hline Female (19-50) & average $r$ & $\min$ & $\max$ & average & $\min$ & $\max$ & average & $\min$ & $\max$ & average & $\min$ & $\max$ \\
\hline f\&v w/ juice & 1.667228 & 1.406116 & 2.226803 & 1.567316 & 1.356022 & 2.152147 & 2.032832 & 1.711176 & 2.720676 & 2.065936 & 1.748086 & 2.764148 \\
\hline grain & 0.750926 & 0.750926 & 0.750926 & 0.785141 & 0.785141 & 0.785141 & 1.003489 & 1.003489 & 1.003489 & 1.067015 & 1.067015 & 1.067015 \\
\hline milk & 1.870727 & 1.870727 & 1.870727 & 1.835318 & 1.835318 & 1.835318 & 2.039818 & 2.039818 & 2.039818 & 1.939273 & 1.939273 & 1.939273 \\
\hline meat & 1.069365 & 0.910374 & 1.331968 & 1.030724 & 0.882027 & 1.312339 & 1.473821 & 1.186196 & 1.834478 & \begin{tabular}{|l}
3.554359 \\
\end{tabular} & 1.274881 & 1.9721 \\
\hline Total Cost (Daily) & 5.358246 & 4.938143 & 6.180424 & 5.218498 & 4.858508 & 6.084945 & 6.54996 & 5.940679 & 7.59846 & 6.626582 & 6.029254 & 7.742535 \\
\hline f\&v w/ juice & 2.111822 & 1.78108 & 2.820617 & 1.985266 & 1.717628 & 2.726053 & \begin{tabular}{|l}
3.57492 \\
\end{tabular} & 2.167489 & 3.446189 & 2.616852 & 2.214242 & 3.501254 \\
\hline grain & 0.578839 & 0.578839 & 0.578839 & 0.605213 & 0.605213 & 0.605213 & 0.773523 & 0.773523 & 0.773523 & 0.822491 & 0.822491 & 0.822491 \\
\hline protein & 2.88462 & 2.639509 & 3.289466 & 2.801653 & 2.572412 & 3.235811 & 3.619878 & 3.176457 & 4.17589 & 3.677609 & 3.246747 & 4.321626 \\
\hline Total Cost (Daily) & 5.575281 & 4.999428 & \begin{tabular}{|l|}
6.688923 \\
\end{tabular} & 5.392132 & 4.895252 & 6.567076 & 6.968321 & 6.117468 & 8.395602 & 7.116951 & 6.283479 & 8.64537 \\
\hline & & & & & & & & & & & & \\
\hline & May-07 & & & Dec-07 & & & May-18 & & & Dec-18 & & \\
\hline Male Teen(14-18) & average & $\min$ & $\max$ & average & $\min$ & $\max$ & average & $\min$ & $\max$ & average & $\min$ & $\max$ \\
\hline f\&v w/ juice & 1.778377 & 1.499857 & 2.375257 & 1.671803 & 1.446423 & 2.295623 & $\begin{array}{ll}3 & 2.168354\end{array}$ & 1.825254 & 2.902054 & 2.203665 & 1.864625 & 2.948425 \\
\hline grain & 0.87608 & 0.87608 & $\begin{array}{l}3 \\
\end{array}$ & 0.915998 & 0.915998 & 0.915998 & $\begin{array}{ll}3 & 1.170737\end{array}$ & 1.170737 & 1.170737 & 1.244851 & 1.244851 & 1.244851 \\
\hline milk & 1.870727 & 1.870727 & 1.870727 & 1.835318 & 1.835318 & 1.835318 & 2.039818 & 2.039818 & 2.039818 & 1.939273 & 1.939273 & 1.939273 \\
\hline meat & 2.138729 & 1.820748 & 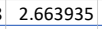 & 2.061447 & 1.764054 & $\begin{array}{r}2.624679 \\
\end{array}$ & 2.947643 & 2.372393 & 3.668955 & 3.108718 & $\begin{array}{l}3 \\
\end{array} .549762$ & 3.944199 \\
\hline Total Cost (Daily) & 6.663913 & 6.067412 & 7.786 & 6.484566 & 5.961793 & 7.671618 & 8.326552 & 7.408202 & 9.781565 & 8.496506 & 7.59851 & 10.07675 \\
\hline f\&v w/ juice & 2.389694 & 2.015433 & 3.191751 & 2.246486 & 1.943631 & 3.084744 & 2.913726 & 2.452685 & 3.899635 & 2.961174 & 2.505589 & 3.961946 \\
\hline grain & 0.672704 & 0.672704 & 0.672704 & 0.703355 & 0.703355 & 0.703355 & 0.898959 & 0.898959 & 0.898959 & 0.955867 & 0.955867 & 0.955867 \\
\hline protein & 3.352396 & 3.067538 & 3.822893 & 3.255975 & 2.98956 & 3.760537 & 4.206886 & 3.691558 & 4.853062 & 4.273978 & 3.773246 & 5.02243 \\
\hline Total Cost (Daily) & 6.414794 & 5.755675 & 7.687349 & 6.205816 & 5.636547 & 7.548636 & 8.01957 & 7.043201 & 9.651655 & 8.19102 & 7.234703 & 9.940243 \\
\hline & May-07 & & & Dec-07 & & & May-18 & & & Dec-18 & & \\
\hline Female Child (9-13) & average & $\min$ & $\max$ & average & $\min$ & $\max$ & average & $\min$ & $\max$ & average & $\min$ & $\max$ \\
\hline f\&v w/ juice & 1.333783 & 1.124893 & 1.781443 & 1.253852 & 1.084817 & 1.721717 & 1.626265 & 1.36894 & 2.17654 & 1.652749 & 1.398469 & 2.211319 \\
\hline grain & 0.750926 & 0.750926 & 0.750926 & 0.785141 & 0.785141 & 0.785141 & 1.003489 & 1.003489 & 1.003489 & 1.067015 & 1.067015 & 1.067015 \\
\hline milk & 1.870727 & 1.870727 & 1.870727 & 1.835318 & 1.835318 & 1.835318 & 2.039818 & 2.039818 & 2.039818 & 1.939273 & \begin{tabular}{|l}
3.939273 \\
\end{tabular} & 1.939273 \\
\hline meat & 1.069365 & 0.910374 & 1.331968 & 1.030724 & 0.882027 & 1.312339 & 1.473821 & 1.186196 & 1.834478 & 1.554359 & 1.274881 & 1.9721 \\
\hline Total Cost (Daily) & 5.0248 & 4.65692 & 5.735063 & 4.905035 & 4.587303 & 5.654516 & 6.143394 & 5.598444 & 7.054325 & 6.213395 & 5.679637 & 7.189706 \\
\hline f\&v w/ juice & 1.889525 & 1.593598 & 2.52371 & 1.776291 & 1.536825 & 2.4391 & 2.303876 & 1.939332 & 3.083432 & 2.341394 & + 1.981164 & 3.132701 \\
\hline grain & 0.531906 & 0.531906 & 0.531906 & 0.556141 & 0.556141 & 0.556141 & 0.710805 & 0.710805 & 0.710805 & 0.755802 & 0.755802 & 0.755802 \\
\hline protein & 2.650732 & 2.425495 & 3.022753 & 2.574492 & 2.363838 & 2.973448 & 3.326375 & 2.918906 & 3.837305 & 3.379424 & +2.983497 & 3.971223 \\
\hline Total Cost (Daily) & 5.072163 & 4.550999 & 6.078369 & 4.906924 & 4.456804 & 5.968689 & 6.341055 & 5.569043 & 7.631541 & 6.47662 & 5.720463 & 7.859727 \\
\hline & & & & & & & & & & & & \\
\hline & & & & & & & & & & & & \\
\hline Daily & May-07 & & & Dec-07 & & & May-18 & & & Dec-18 & & \\
\hline Family of 4 Daily food & & & & & & & & & & & & \\
\hline costs** & average & $\min$ & $\max$ & average & $\min$ & $\max$ & average & $\min$ & $\max$ & average & $\min$ & $\max$ \\
\hline 2007 Proportions & 23.25658 & 21.24078 & 27.10781 & 22.64593 & 20.89449 & 26.71394 & 28.91054 & 25.87672 & 33.87171 & 29.45517 & 26.48571 & 34.80101 \\
\hline 2019 Proportions & 23.62621 & 21.19563 & 28.32077 & 22.85501 & 20.75623 & 27.80859 & 29.53502 & 25.93671 & 35.55491 & 30.1661 & 26.6416 & 36.61675 \\
\hline Difference (2019-2007) & 0.36963 & -0.04515 & 1.212958 & 0.209078 & -0.13826 & 1.094645 & 0.624476 & 0.059985 & 1.683199 & 0.710933 & 0.155889 & 1.815743 \\
\hline Adjusted Difference * & 0.406593 & -0.04967 & 1.334253 & 0.229985 & -0.15208 & \begin{tabular}{|l|}
3.20411 \\
\end{tabular} & 0.686923 & 0.065984 & 1.851519 & 0.782026 & 0.171478 & \begin{tabular}{|l|l|} 
& 1.997318 \\
\end{tabular} \\
\hline dantered to the prop & a dicea $\mathrm{b}$ & by the 2019 & (910 & verage & e, it wou & De & 20071 & asive every da & day & & & th \\
\hline * Added $10 \%$ to all cal & 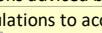 & ccount for $\mathrm{cc}$ & & & 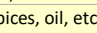 & & & & & & & \\
\hline **Assumption: $\mathrm{Fa}$ & & in & & S & & & & & & & & \\
\hline & & & & & & & & & & & & \\
\hline Weekly & May-07 & & & Dec-07 & & & May-18 & & & Dec-18 & & \\
\hline $\begin{array}{l}\text { Family of } 4 \text { Daily food } \\
\text { costs** }\end{array}$ & average & $\min$ & $\max$ & average & $\min$ & $\max$ & average & $\min$ & $\max$ & average & $\min$ & $\max$ \\
\hline 2007 Proportions & 162.7961 & 148.6855 & 189.7547 & 158.5215 & 146.2614 & 186.9976 & 202.3738 & 181.1371 & 237.102 & 206.1862 & 185.4 & 243.6071 \\
\hline 2019 Proportions & 165.3835 & 148.3694 & 198.2454 & 159.9851 & 145.2936 & 194.6601 & 206.7451 & 181.557 & 248.8844 & 211.1627 & 186.4912 & 256.3173 \\
\hline Difference (2019-2007) & 2.587409 & -0.31607 & 8.490703 & 1.463543 & -0.9678 & 7.662517 & 4.37133 & 0.419895 & 11.78239 & 4.976528 & 1.091221 & 12.7102 \\
\hline Adjusted Difference * & 2.84615 & -0.34768 & 9.339773 & 1.609898 & -1.06459 & 8.428769 & 4.808463 & 0.461885 & 12.96063 & 5.474181 & 1.200343 & 13.98122 \\
\hline
\end{tabular}

Using the results of this study to budget for food would be incorrect, the data used to calculate is not comprehensive enough to account for all food a family would buy Note: data used is not comprehensive enough, there are other foods recommended by both food guide that are not accounted for due to a lack of data availability 
Table 3. Plate details, weighed data, family composition and conversions for 2019 guide

\begin{tabular}{|c|c|c|c|c|c|c|c|c|c|c|c|c|}
\hline & 2007 & MIN & MAX & 07 & MIN & MAX & & MIN & MAX & 02018 & MIN & MAX \\
\hline Aver & 91 & 606916 & 887978 & 687149 & 8018 & 874893 & 982548 & 98 & 222985 & 036239 & .84 & \\
\hline At & & & & & & & & & & & 77 & 95 \\
\hline Average F\&V & 9 & 0.130497 & 705 & 0.165 & 76 & 76 & 0.23 & 0.17 & 0.37 & 54 & 96 & 0.3 \\
\hline verage $F \& V w /$ & 0.222297 & 0.187482 & 0.296907 & 0.208975 & 0.180803 & 0.286953 & 0.271044 & 0.228157 & 0.362757 & 0.275458 & 0.233078 & 0.3685 \\
\hline
\end{tabular}



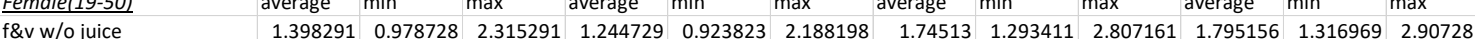
\begin{tabular}{|l|l|l|l|l|l|l|l|l|l|l|l|l|l|l|l|l|l|l|}
\hline grain & 0.750926 & 0.750926 & 0.750926 & 0.785141 & 0.785141 & 0.785141 & 1.003489 & 1.003489 & 1.003489 & 1.067015 & 1.067015 & 1.067015 \\
\hline
\end{tabular} \begin{tabular}{|l|l|l|l|l|l|l|l|l|l|l|l|l|l|l|}
\hline protein & 2.800447 & 2.497608 & 3.300643 & 2.712393 & 2.42916 & 3.248803 & 3.639858 & 3.092001 & 4.326822 & 3.752224 & 3.219885 & 4.54792 \\
\hline
\end{tabular}

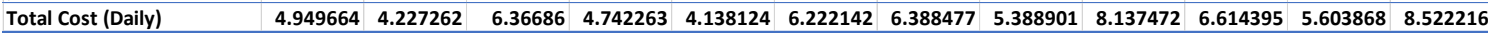



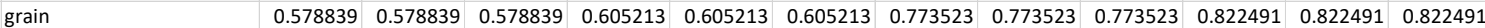
\begin{tabular}{|l|l|l|l|l|l|l|l|l|l|l|l|l|l|l|}
\hline & 2.590421 & 4.128849 & 6.564636 & 4.690833 & 4.022362 & 6.382073 & 6.350889 & 5.271944 & 8.331571 & 6.567162 & 5.469044 & 8.711873 \\
\hline
\end{tabular}

\begin{tabular}{|c|c|c|c|c|c|c|c|c|c|c|c|c|}
\hline \multirow[b]{2}{*}{ Teen Male (14-18) } & \multicolumn{2}{|l|}{ May-07 } & \multirow[b]{2}{*}{$\max$} & \multicolumn{2}{|l|}{ Dec-07 } & \multirow[b]{2}{*}{$\max$} & \multicolumn{2}{|l|}{ May-18 } & \multirow[b]{2}{*}{$\max$} & \multicolumn{2}{|l|}{ Dec-18 } & \multirow[b]{2}{*}{$\max$} \\
\hline & average & $\min$ & & average & $\min$ & & average & $\min$ & & average & $\min$ & \\
\hline f\&v w/o juice & 1.49151 & 1.043977 & 2.469644 & 1.327711 & 0.985411 & 2.334078 & 1.861472 & 1.379639 & 2.994305 & 1.914833 & 1.404767 & 3.1011 \\
\hline grain & 0.87608 & 0.87608 & 0.87608 & 0.915998 & 0.915998 & 0.915998 & 1.170737 & 1.170737 & 1.170737 & 1.244851 & 1.244851 & 1.244851 \\
\hline protein & 3.640581 & 3.24689 & 4.290836 & 3.52611 & 3.157909 & 4.223444 & 4.731815 & 4.019601 & 5.624869 & 4.877891 & 4.18585 & 5.912296 \\
\hline Total Cost (Daily) & 6.008172 & 5.166947 & 7.63656 & 5.769819 & 5.059317 & 7.47352 & 7.764024 & 6.569977 & 9.789911 & 8.037575 & 6.835467 & 10.25825 \\
\hline f\&v w/o juice & 2.004217 & 1.402844 & 3.318584 & 1.784112 & 1.324146 & 3.136417 & 2.501353 & 1.85389 & 4.023598 & 2.573057 & 1.887655 & 4.167103 \\
\hline grain & 0.672704 & 0.672704 & 0.672704 & 0.703355 & 0.703355 & 0.703355 & 0.898959 & 0.898959 & 0.898959 & 0.955867 & 0.955867 & 0.955867 \\
\hline protein & 3.01048 & 2.684928 & 3.548192 & 2.915822 & 2.611347 & 3.492464 & 3.912847 & 3.323901 & 4.651334 & 4.033641 & 3.461376 & 4.889014 \\
\hline \multirow[t]{2}{*}{ Total Cost (Daily) } & 5.687402 & 4.760477 & 7.53948 & 5.403289 & 4.638849 & 7.332236 & 7.313159 & 6.076749 & 9.573891 & 7.562565 & 6.304898 & 10.01198 \\
\hline & May-07 & & & Dec-07 & & & May-18 & & & Dec-18 & & \\
\hline Female Child (9-13) & average & $\min$ & $\max$ & average & $\min$ & $\max$ & average & $\min$ & $\max$ & average & $\min$ & $\max$ \\
\hline f\&v w/o juice & 1.118633 & 0.782983 & 1.852233 & 0.995784 & 0.739059 & 1.750559 & 1.396104 & 1.034729 & 2.245729 & 1.436125 & 1.053575 & 2.325825 \\
\hline grain & 0.750926 & 0.750926 & 0.750926 & 0.785141 & 0.785141 & 0.785141 & 1.003489 & 1.003489 & 1.003489 & 1.067015 & 1.067015 & 1.067015 \\
\hline protein & 2.800447 & 2.497608 & 3.300643 & 2.712393 & 2.42916 & 3.248803 & 3.639858 & 3.092001 & 4.326822 & 3.752224 & 3.219885 & 4.54792 \\
\hline Total Cost (Daily) & 4.670006 & 4.031516 & 5.903802 & 4.493317 & 3.95336 & 5.784503 & 6.039451 & 5.130219 & 7.57604 & 6.255364 & 5.340474 & 7.94076 \\
\hline f\&v w/o juice & 1.58473 & 1.109226 & 2.623996 & 1.410693 & 1.047 & 2.479958 & 1.977814 & 1.465866 & 3.18145 & 2.03451 & 1.492565 & 3.294919 \\
\hline grain & 0.531906 & 0.531906 & 0.531906 & 0.556141 & 0.556141 & 0.556141 & 0.710805 & 0.710805 & 0.710805 & 0.755802 & 0.755802 & 0.755802 \\
\hline protein & 2.38038 & 2.122967 & 2.805547 & 2.305534 & 2.064786 & 2.761483 & 3.093879 & 2.628201 & 3.677799 & 3.18939 & 2.736902 & 3.865732 \\
\hline Total Cost (Daily) & 4.497016 & 3.764098 & 5.961449 & 4.272368 & 3.667927 & 5.797582 & 5.782498 & 4.804871 & 7.570053 & 5.979703 & 4.985269 & 7.916453 \\
\hline
\end{tabular}

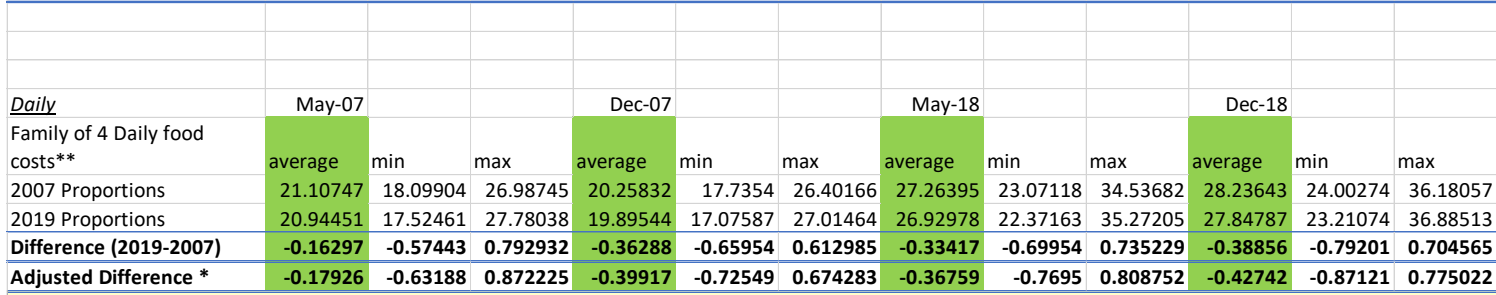

Interpreting results: In May 2007, if a family of 4 ate based on the food recommended by the 2019 food guide, it would make them less food insecure if they adhered to the proportions adviced by the 2019 food guide, on average, it would be $\$ 1.30$ less expensive every day

\begin{tabular}{|c|c|c|c|c|c|c|c|c|c|c|c|c|}
\hline Weekly & May-07 & & & Dec-07 & & & May-18 & & & Dec-18 & & \\
\hline $\begin{array}{l}\text { Family of } 4 \text { Daily food } \\
\text { costs** }\end{array}$ & average & $\min$ & $\max$ & average & $\min$ & $\max$ & average & $\min$ & $\max$ & average & $\min$ & $\max$ \\
\hline 2007 Proportions & 147.7523 & 126.6933 & 188.9121 & 141.8082 & 124.1478 & 184.8116 & 190.8477 & 161.4982 & 241.7578 & 197.655 & 168.0192 & 253.264 \\
\hline Difference (2019-2007) & -1.14076 & -4.02103 & 5.550521 & -2.54018 & -4.61675 & 4.290894 & -2.33921 & -4.89679 & 5.146602 & -2.71993 & -5.54405 & 4.931956 \\
\hline Adjusted Difference * & -1.25484 & -4.42313 & 6.105573 & -2.7942 & -5.07843 & 4.719983 & -2.57313 & -5.38647 & 5.661262 & -2.99193 & -6.09846 & 5.425152 \\
\hline
\end{tabular}


The 'plates' were used to estimate the minimum, the average, and the maximum cost per day of feeding a family of four daily. This family of four consists of an adult male and female between the age of 19-50, a teen male between the age of 14-18, and a female child between 9-13 years and is based on the methodology used by public health units across Canada in creating their Nutritious Food Baskets (Proof Food Insecurity Policy Research, 2018). For further detail on proportions of food, refer to Table 4.

Table 4. Comparative Food Proportions for a Family of Four based on 2007 and 2019 Food Guide Recommended Servings

\begin{tabular}{|c|c|c|c|}
\hline \multicolumn{4}{|l|}{2007 Food Guide } \\
\hline Food Category & $\begin{array}{l}\text { Recommended } \\
\text { servings }\end{array}$ & $\begin{array}{l}\text { Proportion of } \\
\text { Daily Intake }\end{array}$ & $\begin{array}{l}\text { Percentage of } \\
\text { Daily Intake }\end{array}$ \\
\hline \multicolumn{4}{|l|}{ Males (Aged 19-50) } \\
\hline Vegetables and Fruit & $8-10$ (average 9 ) & $9 / 22$ & $40 \%$ \\
\hline Grains & 8 & $8 / 22$ & $36.5 \%$ \\
\hline Milk and Alternatives & 2 & $2 / 22$ & $9 \%$ \\
\hline Meat and Alternatives & 3 & $3 / 22$ & $13.5 \%$ \\
\hline \multicolumn{4}{|l|}{ Females (Aged 19-50) } \\
\hline Vegetables and Fruit & 7-8 (Average 7.5$)$ & $7.5 / 18.5$ & $40.5 \%$ \\
\hline Grains & 6 & $6 / 18.5$ & $32.5 \%$ \\
\hline Milk and Alternatives & 3-4 (Average 3.5) & $3.5 / 18.5$ & $19 \%$ \\
\hline Meat and Alternatives & $1-2$ (Average 1.5$)$ & $1.5 / 18.5$ & $8 \%$ \\
\hline \multicolumn{4}{|c|}{ Male Teen (Aged 14-18) } \\
\hline Vegetables \& Fruit & 8 & $8 / 21.5$ & $37.21 \%$ \\
\hline Grains & 7 & $7 / 21.5$ & $32.56 \%$ \\
\hline Milk and Alternatives & 3-4 (Average 3.5) & $3.5 / 21.5$ & $16.28 \%$ \\
\hline Meat and Alternatives & 3 & $3 / 21.5$ & $13.95 \%$ \\
\hline \multicolumn{4}{|c|}{ Female Child (Aged 9-13) } \\
\hline Vegetables and Fruit & 6 & $6 / 17$ & $35.25 \%$ \\
\hline Grains & 6 & $6 / 17$ & $35.25 \%$ \\
\hline Milk and Alternatives & 3-4 (Average 3.5) & $3.5 / 17$ & $20.5 \%$ \\
\hline Meat and Alternatives & $1-2$ (Average 1.5$)$ & $1.5 / 17$ & $9 \%$ \\
\hline \multicolumn{4}{|c|}{ TOTALS (family of four) } \\
\hline Vegetables and Fruit & - & $30.5 / 79$ & - \\
\hline Grains & - & $27 / 79$ & - \\
\hline Milk and Alternatives & - & $12.5 / 79$ & - \\
\hline Meat and Alternatives & - & 9/79 & - \\
\hline \multicolumn{4}{|l|}{2019 Food Guide } \\
\hline \multicolumn{4}{|l|}{ Males (Aged 19-50) } \\
\hline Vegetables and Fruit & 11 & $11 / 22$ & $50 \%$ \\
\hline Protein Foods & 5.5 & $5.5 / 22$ & $25 \%$ \\
\hline Whole Grain Foods & 5.5 & $5.5 / 22$ & $25 \%$ \\
\hline \multicolumn{4}{|c|}{ Females (Aged 19-50) } \\
\hline Vegetables and Fruit & 9.25 & $9.25 / 18.5$ & $50 \%$ \\
\hline Protein Foods & 4.625 & $4.625 / 18.5$ & $25 \%$ \\
\hline Whole Grain Foods & 4.625 & $4.625 / 18.5$ & $25 \%$ \\
\hline \multicolumn{4}{|c|}{ Male Teen (Aged 14-18) } \\
\hline Vegetables and Fruit & 10.75 & $10.75 / 21.5$ & $50 \%$ \\
\hline Protein Foods & 5.375 & $5.375 / 21.5$ & $25 \%$ \\
\hline Whole Grain Foods & 5.375 & $5.375 / 21.5$ & $25 \%$ \\
\hline \multicolumn{4}{|c|}{ Female Child (Aged 9-13) } \\
\hline Vegetables and Fruit & 8.5 & $8.5 / 17$ & $50 \%$ \\
\hline Protein Foods & 4.25 & $4.25 / 17$ & $25 \%$ \\
\hline Whole Grain Foods & 4.25 & $4.25 / 17$ & $25 \%$ \\
\hline \multicolumn{4}{|c|}{ TOTALS (family of four) } \\
\hline Vegetables and Fruit & - & $39.5 / 79$ & - \\
\hline Protein Foods & - & $19.75 / 79$ & - \\
\hline Whole Grain Foods & - & $19.75 / 79$ & - \\
\hline
\end{tabular}


We then costed the total number of food servings needed to meet CFG recommendations based on both 2007 and 2019 food prices. These scenarios serve to develop and compare the cost of food from both Food Guides based on prices in 2007 and 2019 and are described in more detail below.

1. Average retail prices of food recommended by the 2007 Food Guide and costed at 2007 food prices.

2. Average retail prices of food recommended by the 2007 Food Guide and costed at 2019 food prices.

3. Average retail prices of foods recommended by the 2019 Food Guide and costed at 2007 food prices.

4. Average retail prices of foods recommended by the 2019 Food Guide and costed at 2019 food prices.

The food prices came from a list of 27 retail prices of foods found in CANSIM 360-0012 (Statistics Canada, 2019). Retail prices for selected foods are collected in supermarkets as well as drugstores on a regular monthly basis. Average prices of each product are weighed by the population of the area in question to calculate the average Canadian retail prices of each product.

Using the list of 27 different food products, the retail prices of May 2007 and May 2018 were collected. The month of May was chosen to represent a year's food costs because food prices in May are considered not to be affected by "high availability of food from the fall harvest or mid-winter food transportation costs" (Proof Food Insecurity Policy Research, 2018). These prices are collated into lists based on their inclusion in the 2007 and/or 2019 Food Guides (i.e. a food may be recommended by only one Food Guide, such as fruit juice, or by both Food Guides, such as apples). From this list of recommended foods and their associated prices, the foods are grouped into their corresponding food groups based on their Food Guide. This meant there were four food groupings for the 2007 CFG and only three food groupings for the 2019 CFG (see Table 4).

The average price of all the food items in these food groups was calculated and then divided to create one serving of that item (i.e., two eggs or $75 \mathrm{~g}$ of meat). We then used these cost estimates of one serving of each food group based on the 2007 or 2019 Food Guides to calculate the cost of a full plate of food. For example, with the average price of one serving of the 2007 CFG meat and alternatives food group, we calculated the total meat and alternative servings a family of four would need in a day, and estimated the cost of this food group (see Table 1 for these totals). We repeated this for all food groups for both the 2007 and 2019 CFG using retail prices from May 2007 and May 2018.

It is important to note some assumptions made when creating the costs of these plates. When converting the quantities of the retail prices of food, we assume that there is no food loss. In other words, if 1000 grams of food are purchased, exactly 1000 grams of food can be consumed; there is no plate loss or food dropped during preparation. Additionally, we assume that the purchased food is equivalent to cooked food. This means that if, for instance, one requires 75 grams of ground beef for one serving of protein, they will need to purchase exactly 75 grams of ground beef. We also added $10 \%$ to all calculations to account for the cost of miscellaneous food items, such as oils, spices, etc. and assumed that the family can cook and prepare all their daily and weekly meals at home.

\section{Results}

The demographic of our survey population is reflective of the Canadian population (see Table 5). 
Table 5. Demographics of Survey Respondents

\begin{tabular}{|c|c|}
\hline \multicolumn{2}{|l|}{ Survey Respondent Demographics $(\mathrm{n}=1017)$} \\
\hline Descriptor & \% of respondents \\
\hline Gender & \\
\hline Male & $46 \%$ \\
\hline Female & $52 \%$ \\
\hline Non-binary & $1 \%$ \\
\hline Prefer not to answer & $1 \%$ \\
\hline Age & \\
\hline $18-29$ years old & $17 \%$ \\
\hline $30-39$ years old & $21 \%$ \\
\hline $40-49$ years old & $22 \%$ \\
\hline $50-59$ years old & $21 \%$ \\
\hline $60-69$ years old & $15 \%$ \\
\hline $70-79$ years old & $4 \%$ \\
\hline 79 or older & $0 \%$ \\
\hline Highest completed school degree & \\
\hline Less than high school diploma & $3 \%$ \\
\hline High school diploma or equivalent & $24 \%$ \\
\hline Some college or university, no degree & $22 \%$ \\
\hline College diploma & $23 \%$ \\
\hline Bachelor's degree & $19 \%$ \\
\hline Master's degree & $6 \%$ \\
\hline Professional degree & $3 \%$ \\
\hline Doctorate & $1 \%$ \\
\hline Household income & \\
\hline$<\$ 40,000$ & $34 \%$ \\
\hline$\$ 40,001-\$ 80,000$ & $33 \%$ \\
\hline$\$ 80,001-\$ 150,000$ & $26 \%$ \\
\hline$>\$ 150,001$ & $7 \%$ \\
\hline Province & \\
\hline Atlantic & $9 \%$ \\
\hline Quebec & $20 \%$ \\
\hline Ontario & $41 \%$ \\
\hline Prairies & $15 \%$ \\
\hline British Columbia & $12 \%$ \\
\hline Territories & \\
\hline Employment Status & Data not available. \\
\hline Employed full time ( 30 or more hours per week) & \\
\hline Employed part time (up to 30 hours per week) & \\
\hline Unemployed and currently looking for work & \\
\hline Unemployed and not currently looking for work & \\
\hline Student & \\
\hline Retired & \\
\hline Homemaker & \\
\hline Self-employed & \\
\hline Unable to work & \\
\hline Other & \\
\hline Dietary preferences* & $6.90 \%$ \\
\hline No dietary preferences & $72.10 \%$ \\
\hline Specific dietary preference & \\
\hline Flexitarian & $5.01 \%$ \\
\hline Vegetarian & $2.06 \%$ \\
\hline Pescatarian & $1.87 \%$ \\
\hline Lacto-ovo Vegetarian & $1.38 \%$ \\
\hline Vegan & $1.27 \%$ \\
\hline Religious/cultural & $1.57 \%$ \\
\hline
\end{tabular}




\subsection{Sources of Nutrition Information}

Overall, CFG does not rank high as a preferred source of information for nutrition advice (see Figure 1). A total of $19.6 \%$ chose family and friends, the most popular option, followed by general research, social media, cookbooks, TV programs, and documentaries. Based on the choices given, the CFG ranked sixth. Health professionals like doctors and nutritionists ranked seventh, with $7.8 \%$ of respondents choosing them as their preferred source. Additionally, there were generational differences in how respondents ranked sources of health information as younger respondents were more likely to turn to celebrities and social media, while older generations looked to cookbooks, self-help books, and magazines (data not shown).



Figure 1. Sources of nutrition-relation information

\subsection{Awareness and Use of the Food Guide}

Awareness of CFG was high among our survey respondents (91.4\%), and over 74\% were familiar with the new CFG released a few months earlier. When looking at the demographics of our population, women had a higher awareness of CFG (95\%) compared to men (89\%). Boomers (aged 25-40 years old) were most likely to be aware that a new CFG was published (80\%) while GenZs (aged 24 years and younger) were least aware (61\%) of the 2019 publication. Results showed regional differences as well. The Atlantic Region was most likely to be aware that a new CFG was published (84\%), and Ontario residents were the least aware (71\%).

In addition, the use of the Food Guide for healthy eating advice varied between different dietary preference groups. Vegetarians, consumers with religious and/or cultural preferences, and flexitarians were most likely to use the Food Guide. In addition, both education and income are strong determinants for awareness and usage of the CFG.

\subsection{Barriers to Implementing Recommendations}

Over $47 \%$ of survey respondents stated they did not face any barriers in adopting CFG recommendations in their daily eating habits. However, the $52.4 \%$ of respondents that do face challenges identified affordability, a lack of compatibility with taste or dietary preferences, and the belief that recommendations are too time-consuming as their main barriers (see Figure 2). Men were more likely than women to indicate that recommendations are too time-consuming, but women were more likely to indicate that recommendations did not meet their dietary needs. The most significant barrier to adoption identified among all regional groups was that recommendations are not affordable. 
Recommended foods are not available to purchase in my area (5.1\%)

Recommendations don't fit my cultural food preferences $(5.5 \%)$

Recommendations are too time consuming (9.5\%)

Recommendations don't fit my dietary needs (10.2\%)

Recommendations don't fit my taste preferences (20.2\%)

Recommendations are not affordable (26.5\%)

I do not face barriers to adoption (47.6\%)

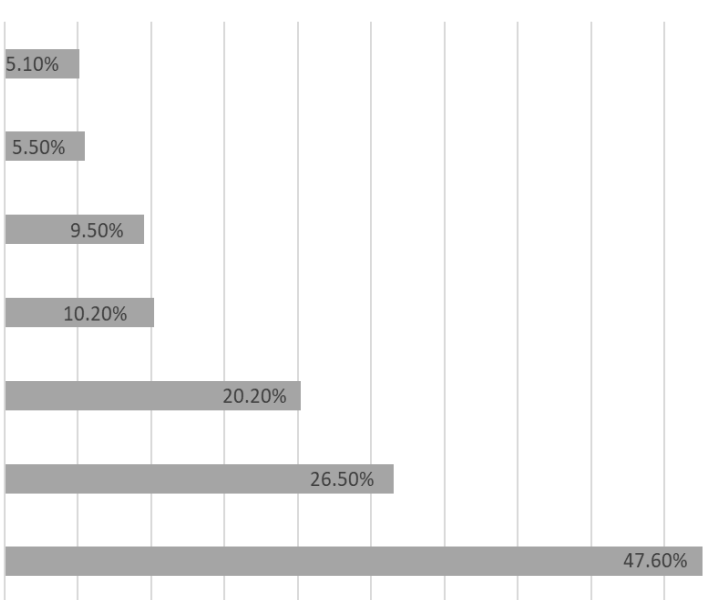

Figure 2. Barriers to adopting Canada's Food Guide recommendations

\subsection{Applicability and Practicality of the Food Guide}

In total, $47 \%$ of respondents believe that the new CFG (2019) reflects their understanding of what constitutes a healthy diet and $49 \%$ believe the Food Guide is an important document that influences food-related behaviours. Again, there were generational differences as Boomers were most likely to disagree or strongly disagree that CFG provides realistic and practical dietary advice and believe that it is an important document for food-related behaviours. Education is also a noteworthy determinant. Respondents with a bachelor's degree were most likely to agree or strongly agree that the new Food Guide is based on scientific evidence and best practices (64\%).

\subsection{Affordability of the Food Guide}

Based on our calculations, the 2019 CFG is less expensive for an average family of 4 (see Figure 3). The 2007 and 2019 Canadian Food Guides recommend different proportions of foods and different types of food. Figure 3 outlines various scenarios for the average daily cost of food for a family of 4 based on the recommended proportions and recommended food types from both the 2007 and 2019 guides.

Figure 3

\begin{tabular}{|c|c|c|c|c|}
\hline \multirow{3}{*}{$\begin{array}{l}\text { Proportion of } \\
\text { Food } \\
\text { Recommended } \\
\text { by } 2007 \text { Food } \\
\text { Guide }\end{array}$} & \multicolumn{2}{|c|}{ Food recommended by 2007 Food Guide } & \multicolumn{2}{|c|}{ Food recommended by 2019 Food Guide } \\
\hline & May 2007 & May 2018 & May 2007 & May 2018 \\
\hline & $\$ 23.26$ & $\$ 28.91$ & $\$ 21.11$ & $\$ 27.26$ \\
\hline \multirow{2}{*}{$\begin{array}{l}\text { Proportion of } \\
\text { Food } \\
\text { Recommended } \\
\text { by } 2019 \text { Food } \\
\text { Guide }\end{array}$} & May 2007 & May 2018 & May 2007 & May 2018 \\
\hline & $\$ 23.63$ & $\$ 29.54$ & $\$ 20.94$ & $\$ 26.93$ \\
\hline
\end{tabular}

The 2007 Food Guide in 2007 and 2018 food prices: In May 2007, eating the food and proportions recommended by the 2007 Food Guide, it would cost a family of four an average of \$23.26 daily. In May 2018, using the same proportions and the same food from the 2007 Food Guide, it would cost $\$ 28.91$ daily. If a family of four wanted to maintain eating the types of food recommended by the 2007 CFG but wanted to change the proportions of the food to those recommended by the new CFG, it would cost them $1.6 \%$ more in 2007 and $2.2 \%$ more if they made this decision in May 2018.

The 2019 Food Guide in 2007 and 2018 food prices: In May 2018, eating the food and proportions 
recommended by the 2019 Food Guide, it would cost a family of four an average of $\$ 26.93$ daily, compared to $\$ 20.94$ at 2007 food prices.

Therefore, when comparing the new 2019 Food Guide to the previous 2007 version, the cost to eat based on the recommendations is more affordable. In today's prices, the $2007 \mathrm{CFG}$ is $\$ 1.98$ per day more expensive than eating based on the 2019 CFG recommended food and proportions.

\section{Discussion}

This is important to consider as dietary choices are heavily influenced by personal preference, religious and cultural beliefs, as well as allergies, which may also impact consumers' ability to meet dietary guidelines if they are too rigid or don't accommodate other cultural styles of eating. For example, the 2007 Food Guide was published in "multiple languages to reflect changing population demographics" and was also adapted to meet the unique cultural needs of First Nations, Inuit, and Metis populations. At the time of this study, these options did not exist yet for the 2019 CFG.

When the new Food Guide was released in January 2019, it garnered tremendous attention in the media, mostly due to the significant changes in the recommendations and visuals of the new CFG but also because of the length of time since an update had been completed. The results from this study and survey confirm that most Canadians have heard of the new Food Guide. The paradox of CFG lies between the awareness, and the importance respondents give to it in relation to how much respondents desire to follow it and give it relevance in their daily lives. There is a fundamental assumption that Food Guides and their recommendations will lead to improved food choices and that food availability will reflect these choices. However, this is only partially correct, and research shows that globally, the impact of guidelines on modifying diets of individuals appears to be small (Anderson \& Zlotkin, 2000).

In addition, Food Guides produced by governments are only one source of healthy eating information for the population and are broad by design to ensure they meet the needs of most of the population (Anderson \& Zlotkin, 2000). It is known that consumers are influenced by several different sources of information when making food choices (Slater \& Mudryj, 2018), and while information and knowledge are mediators of behaviour, they are not enough to create behaviour change. This study adds to the body of literature related to which nutrition information sources consumers of different ages will go to for healthy eating advice. Also, because FBDGs are purposefully broad, they may not be a source of information for consumers who have specific dietary patterns. As stated by Brown et al. (2014), respondents will seek sources that will reinforce previous conventions about dietary patterns and choices. Therefore, prior knowledge acquisition may be causing respondents to be more predisposed to seek evidence that suits their sentiments on dietary choices.

To be effective in bringing about dietary change and achieving health goals, Food Guides should be practical, and the recommended foods should be available, accessible, and affordable (Anderson \& Zlotkin, 2000). When CFG was released, there was little or no evidence that Health Canada conducted a cost analysis on its guide or evaluated the socioeconomics of the guide. Our survey respondents identified affordability as the most important barrier to adopting the recommendations from the Food Guide. Previous surveys also confirm that consumers' top barriers to eating a diet high in fruits and vegetables include cost and access (International Food and Information Council, 2015). Yet, our calculations from Part II of this study show that the 2019 CFG is more affordable. This may not be due to a true change in the price of healthy foods, but more likely because of the changes in quantities and types of recommended food in the 2019 Food Guide. This includes a lower focus and reliance on meat and dairy foods, which typically can be more expensive items at grocery stores. This issue of affordability is timely, given the prevalence of food insecurity in Canada and the lack of significant movement on this outcome in our population. Considering the impact of food and nutrition on health, and the fact that food insecure households are more likely to have health problems and poor diets, affordability should be a factor when developing Food Guides (McIntyre et al., 2016). Previous work in the United States also found that consumers will choose less healthy options due to the costs of healthy alternatives (International Food Information Council Foundation 2018). 30\% of those in fair/poor health said they often chose less healthy options because of the cost (International Food and Information Council, 2015). It will also be important to continue to monitor the affordability of CFG because fruit and vegetable prices are predicted to rise in the future (Charlebois, McCormick, \& Foti, 2017). To our knowledge, this is the first study in Canada that has looked at affordability as a possible barrier to adoption of a food guide.

Some limitations should be considered when interpreting the results of this study. The survey administered for Part I of the study was released eight weeks after the new Food Guide was released. The relatively short period between the release of the Food Guide and the administration of the survey may not have given respondents 
enough time to be exposed to, and therefore understand, the new Food Guide. They may also not have had enough time to fully consider adopting the recommendations or the barriers that may have been preventing them from doing so. Related to Part II of the study: when pricing the quantities of food, it was assumed there was no food loss and that the family of four has time to cook all its meals at home.

\section{Conclusion}

Despite being in existence since 1942, there is little pan-Canadian data looking at the Canadian population's awareness of and adherence to Canada's Food Guide despite small-scale studies that have looked within niche samples or utilized secondary data (Vanderlee, McCrory \& Hammond, 2015; Allen et al., 2011; Slater \& Mudryj, 2018). While Canadians are highly aware of CFG, few believe it to be relevant or important to their dietary habits. In addition, affordability of meeting these recommendations is a concern among Canadians, which is also true when generally looking at food decision-making behaviour. In contrast, our cost analysis demonstrated that eating based on the proportions and foods in the 2019 CFG is more affordable compared to the 2007 version. The process for developing the next recommendations should look to include cost and affordability as these will have important socioeconomic implications as market conditions and food prices change.

However, it's still important to note that Food Guide recommendations are not mandated and are not designed to be followed in their entirety. They serve a useful purpose in establishing population health goals to prevent disease and promote health, provide evidence-based educational resources for teachers to develop lessons for youth and adolescents, and suggest food quantities and options for institutional organizations. But beyond this, our study showed that consumers are not using CFG to make dietary choices, and it is not a preferred source of health information. This is likely due to the multiple sources of health and nutrition information that consumers are exposed to, including print and online sources. In order to effectively influence and change population health, governments should integrate these health and dietary goals with effective partnerships among the many sectors that influence the food supply chain and food selection.

\section{Acknowledgments}

No funding sources were used in the production of those manuscript.

\section{Conflict of interest}

No conflict and/or funding issues to report.

\section{Author Contributions}

"Conceptualization, S.C. S.S. and M.S.; Methodology, S.C., B.N.W. D.F.; Software, D. F.; Validation, S.C., D.F. and J.M.; Formal Analysis, S.C. and B.N.W.; Investigation, M.S.; Resources, J.M.; Data Curation, B.N.W. and D.F.; Writing - Original Draft Preparation, M.S.; Writing - Review \& Editing, S.C., S.S., M.R., I.C., and J.M.; Visualization, S.C and B.N.W.; Supervision, S.C.; Project Administration, J.M.;

\section{References}

Allen, J. P., Taylor, J. G., Rozwadowski, M. M., Boyko, J. A., Blackburn, D. F. (2011). Adherence to Canada's Food Guide among Pharmacy Students. Can. Pharm. J. / Rev. des Pharm. du Canada, 144, 79-84. https://doi.org/10.3821/1913-701X-144.2.79

Anderson, G. H., \& Zlotkin, S. H. (2000). Developing and implementing food-based dietary guidance for fat in the diets of children. Am. J. Clin. Nutr., 72, 1404-1409. https://doi.org/10.1093/ajcn/72.5.1404

Breland, J. Y., McAndrew, L. M., Gross, R. L., Leventhal, H., \& Horowitz, C. R. (2013). Challenges to healthy eating for people with diabetes in a low-income, minority neighborhood. Diabetes Care, 36, 2895-2901. https://doi.org/10.2337/dc12-1632

Brown, K. A., Timotijevic, L., Barnett, J., Shepherd, R., Lähteenmäki, L., \& Raats, M. M. (2011). A review of consumer awareness, understanding and use of food-based dietary guidelines. Br. J. Nutr, 106, 15-26. https://doi.org/10.1017/S0007114511000250

Brug, J. (2008). Determinants of healthy eating: motivation, abilities and environmental opportunities. Fam. Pract., 25, 50-55. https://doi.org/10.1093/fampra/cmn063

Charlebois, S., McCormick, M., \& Foti, L. (2017). Produce Retail Price Volatility and Perceptions in the Canadian Market: Nutrition Security Variances. J. Int. Food Agribus. Mark., 29, 178-196. https://doi.org/10.1080/08974438.2017.1303656

Charlebois, S., McCormick, M., \& Juhasz, M. (2016). Meat consumption and higher prices. Br. Food J., 118, 2251-2270. https://doi.org/10.1108/BFJ-03-2016-0121 
Food-based dietary guidelines. (2019). What are food-based dietary guidelines. Retrieved from http://www.fao.org/nutrition/education/food-dietary-guidelines/background/en/

Gregório, M. J., Rodrigues, A. M., Graça, P., de Sousa, R. D., Dias, S. S., Branco, J. C., Canhão, H. (2018). Food Insecurity Is Associated with Low Adherence to the Mediterranean Diet and Adverse Health Conditions in Portuguese Adults. Front. public Heal., 6, 38. https://doi.org/10.3389/fpubh.2018.00038

Health Canada. (2018). Revision process for Canada's food guide.

International Food and Information Council. (2015). Food and Health Survey 2015.

Loopstra, R., \& Tarasuk, V. (2012). The Relationship between Food Banks and Household Food Insecurity among Low-Income Toronto Families. Can. Public Policy, 38, 497-514. https://doi.org/10.3138/CPP.38.4.497

Love, P., Maunder, E. M. W., \& Green, J. M. (2008). Are South African women willing and able to apply the new food-based dietary guidelines? Lessons for nutrition educators. South African J. Clin. Nutr., 21, 17-24. https://doi.org/10.1080/16070658.2008.11734157

McIntyre, L., Patterson, P. B., Anderson, L. C., \& Mah, C. L. (2016). Household Food Insecurity in Canada: Problem Definition and Potential Solutions in the Public Policy Domain. Can. Public Policy, 42, 83-93. https://doi.org/10.3138/cpp.2015-066

Painter, J., Rah, J.-H., \& Lee, Y.-K. (2002). Comparison of International Food Guide Pictorial Representations. J. Am. Diet. Assoc., 102, 483-489. https://doi.org/10.1016/S0002-8223(02)90113-6

Pelletier, J. E., \& Laska, M. N. (2012). Balancing healthy meals and busy lives: associations between work, school, and family responsibilities and perceived time constraints among young adults. J. Nutr. Educ. Behav., 44, 481-489. https://doi.org/10.1016/j.jneb.2012.04.001

Slater, J. J., \& Mudryj, A. N. (2018). Are we really "eating well with Canada's food guide"? BMC Public Health, 18, 652. https://doi.org/10.1186/s12889-018-5540-4

Statistics Canada. (2019). Monthly average retail prices for food and other selected products.

Tarasuk, V. (2005). Household Food Insecurity in Canada. Toronto, 20. https://doi.org/10.1097/00008486-200510000-00003

The Rome Declaration. (1996). Rome Declaration on World Food Security and World Food Summit Plan of Action. Rome.

Vanderlee, L., McCrory, C., \& Hammond, D. (2015). Awareness and Knowledge of Recommendations from Canada's Food Guide. Can. J. Diet. Pract. Res., 76, 146-149. https://doi.org/10.3148/cjdpr-2015-014

\section{Copyrights}

Copyright for this article is retained by the author(s), with first publication rights granted to the journal.

This is an open-access article distributed under the terms and conditions of the Creative Commons Attribution license (http://creativecommons.org/licenses/by/4.0/). 